\title{
Adhesion Properties of Self-Polymerized Dopamine Thin Film
}

\author{
Fut (Kuo) Yang and Boxin Zhao* \\ Department of Chemical Engineering and Waterloo Institute for Nanotechnology, 200 University Avenue West, \\ Waterloo, Ontario, N2L 3G1, Canada
}

\begin{abstract}
We report an experimental study of the adhesion properties of polydopamine thin films and their bonding behavior on polymer, glass and metal surfaces. Dopamine is able to adsorb onto all surfaces and self-polymerize into a thin hydrophilic film. Dynamic contact angle measurements revealed a large contact angle hysteresis between advancing and receding angles and a possible hydration layer when exposed to water. Polydopamine-coated surfaces in air are relatively inert having a low self-adhesion compared with polydimethylsiloxane elastomer surfaces, reflecting the nonconformal, glassy nature of polydopamine thin films. The dopamine aqueous solution was found able to bond two rigid surfaces (e.g. aluminum and glass) but it might not be suitable for joining soft or flexible polymer surfaces as polydopamine films are glassy and subject to internal cracks induced by the mismatch of elastic modulus. The research findings provide insights into the potential application of dopamine self-polymerization for adhesive bonding or joining of dissimilar materials.
\end{abstract}

Keywords: Polydopamine, dopamine self-polymerization wettability, water contact angle, adhesion and adhesives, joining, adhesive bonding.

\section{INTRODUCTION}

As future technological innovations gear toward miniaturizing machines and maximizing performance density, effective joining of similar or dissimilar material components has become one of the most critical technical prerequisites for manufacturing at ever smaller scales. Compared to soldering and wire bonding technologies, adhesive bonding offers numerous advantages such as environmental friendliness (elimination of lead and flux cleaning), mild processing conditions, fewer processing steps, and especially, the fine pitch capability to make microjoining possible due to the availability of small size conductive fillers. However, the performance of adhesive bonding depends significantly on the quality of surfaces. Surface pretreatments are commonly required for the success of adhesive bonding. In this article, we report an experimental study of the surface and material properties of the self-polymerized polydopamine thin film and discuss its potential application as a novel coating and adhesive material.

Dopamine, commonly known as a neurotransmitter, is a small molecule mimic of the adhesive component, L-DOPA, of marine mussels with the chemical structure of catecholamine. Under alkaline conditions, the catechol functional group oxidizes to quinone allowing dopamine to self-polymerize and form a thin film on support surfaces through covalent bond and other strong intermolecular interactions such as hydrogen-bonding, metal chelation, and $\pi-\pi$ interactions $[1,2]$. In 2007, Lee and coworkers reported

*Address correspondence to this author at the Department of Chemical Engineering and Waterloo Institute for Nanotechnology, 200 University Avenue West, Waterloo, Ontario, N2L 3G1, Canada;

Tel: 519-8884567, Ext. 38666; Fax: 519-7464979;

E-mail: zhaob@uwaterloo.ca the method of dip-coating objects in an aqueous solution of dopamine and found a nanometer thin layer of polydopamine formed on a wide range of different substrates including noble metals, oxides, semiconductors, ceramics, and synthetic polymers [2-4]. They also demonstrated polydopamine coating as a universal route for material functionalization for such applications as improving wet adhesion, conjugating organic components, and supporting spontaneous electroless metal depositions. Lee's work has led to continuously increasing research activities on polydopamine coatings in various fields including biosensors and bioelectronics [5-8], tissue and pharmaceutical engineering [9-12], nanotechnology [13-17], and membrane science [18-24]. Recently, Ryu and coworkers reported dopamine coating as a universal route to produce robust organic-inorganic biocomposites by bio-mineralization of hydroxyapatite on polydopamine thin films [25]. Xing and Yin reported that polydopamine is a suitable adhesive for assembling halloysite nanotubes on electrodes [26]. $\mathrm{Xu}$ and coworkers reported the dopamine-induced reduction and functionalization of graphene oxide nanosheets for subsequent grafting of polymer brushes [27]. Bernsmann and coworkers characterized the dopamine-melanin growth on silicon oxide and found that the thickness of these coatings can be controlled to allow a growth regime that is proportional to reaction time $[22,28]$.

In this study, we hypothesize that dopamine aqueous solutions can be used as adhesives since dopamine adsorbs on almost all kinds of surfaces, in particular on metal surfaces by forming strong coordination bonds [1,29], and develops cohesive strength through self-polymerization [30]. To the authors' best knowledge, there have been no systematic studies of the adhesion and associated micromechanical properties of polydopamine thin films. The research works reported in the article are the first steps toward elucidating the adhesion behavior of polydopamine. Three types of experimental studies (water contact angle, 
contact adhesion, and adhesive bonding) were described and discussed to derive fundamental insights into the wettability and adhesion behavior of polydopamine thin films coated on or bonded between metal and glass or polymer substrates.

\section{MATERIALS AND EXPERIMENTAL}

Dopamine hydrochloride was supplied by SigmaAldrich. TRIS-HCl buffer base (BP152) was supplied by Fisher BioReagents. Polydimethylsiloxane (PDMS, Sylgard 184) was supplied by Dow Corning Corp., Midland, MI. Aluminum SEM studs (Product No: 16111) were supplied by TED PELLA, INC. Plain microscope slides were supplied by Fisher Scientific. Microscope slides and aluminum stools were cleaned by acetone and deionized water before use. The experimental setup is illustrated in Fig. (1) and described below.

\subsection{Preparation of Flat Sheets and Hemispherical Tips of PDMS}

PDMS solution used for making elastomers was prepared by mixing the elastomer base and crosslinker at a weight ratio of 10:1. The flat sheet of PDMS was made by casting 2 $\mathrm{mL}$ of PDMS solution onto a microscope slide and curing at $90{ }^{\circ} \mathrm{C}$ for 1.5 hours in ambient air. The sheet thickness was measured to be $1.25 \pm 0.1 \mathrm{~mm}$. The hemispherical tip of PDMS was made by first molding the PDMS solution into a hemispherical shape using a custom-made Teflon mold and then coating the resulting PDMS tip with a layer of PDMS solution to make the tip surface smooth. The tip core was cured at $90{ }^{\circ} \mathrm{C}$ for $15 \mathrm{~min}$ and the tip coating along with the core was then cured at $90{ }^{\circ} \mathrm{C}$ for 1.5 hours in ambient air. The resulting tip radius was measured to be $2.8 \pm 0.1 \mathrm{~mm}$ by analyzing the side-view image of the tip using a customwritten MATLAB (R2008a, MathWorks) script which gives a least squares best-fit to the curvature of the apex of the tip.

\subsection{Coating Polydopamine Thin Films on PDMS Elastomers}

The dopamine solution used for coating was made by dissolving dopamine hydrochloride at a concentration of 2 $\mathrm{mg} / \mathrm{mL}$ in a $10 \mathrm{mM}$ TRIS-HCl buffer at $\mathrm{pH}$ 8.5. PDMS elastomers were submerged in the dopamine solution immediately for a period of 24 hours once the solution was made. To avoid the deposition of polydopamine nanoparticles formed in the solution during the coating process, all samples of PDMS elastomers were placed upside down in the solution. The coated samples were air dried overnight. The surface quality of the coatings was examined by optical microscope (OmanoOMM300T) and Veeco Optical Profiling System (WYKO NT1100).

\subsection{Contact Angle Measurements of Polydopamine Thin Films}

The sessile drop method was used to determine the wettability of deionized water on surfaces coated in 2 $\mathrm{mg} / \mathrm{mL}$ dopamine solution for 24 hours, as shown in Fig.

(A)

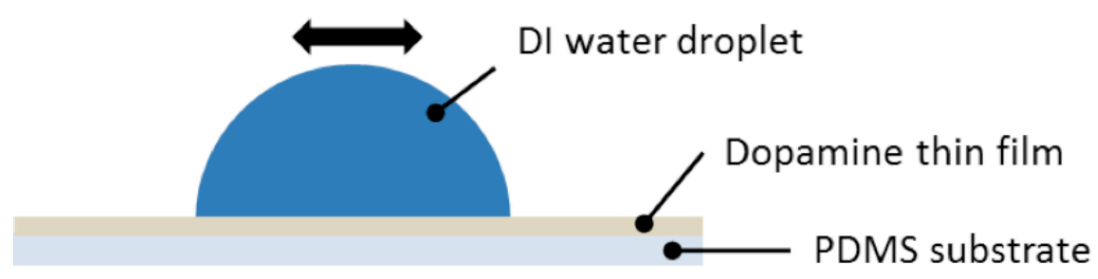

(B)

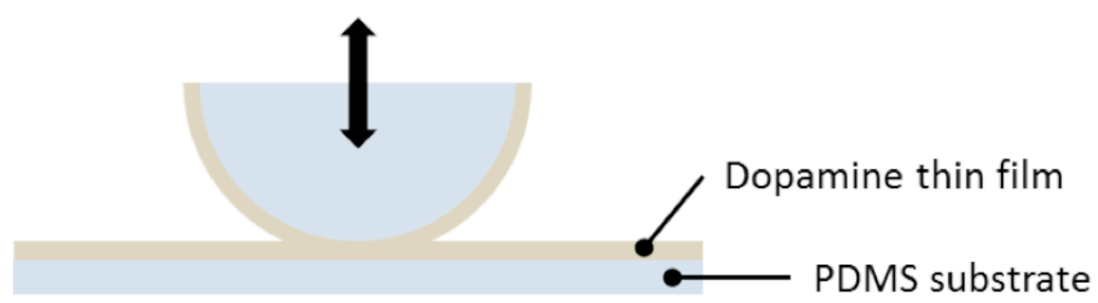

(C)

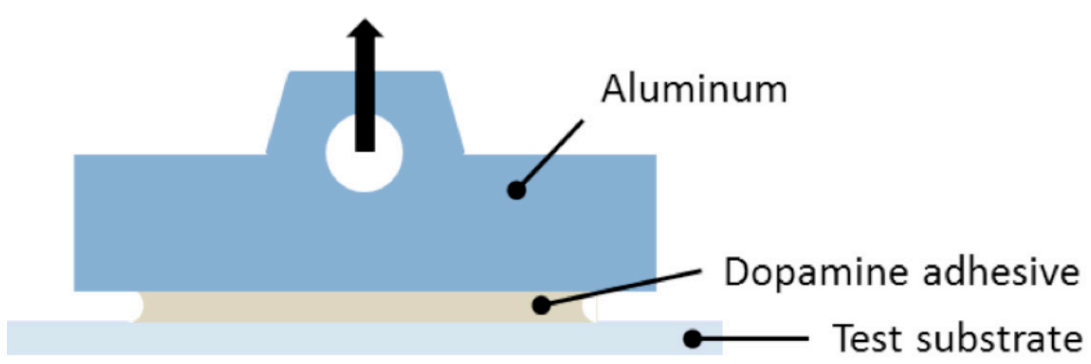

Fig. (1). Schematics of three characterization techniques: (A) contact angle measurement, (B) contact adhesion test, and (C) pull-off bonding test. Black arrows represent the direction of movements. 
(1A). A drop of $5 \mu \mathrm{L}$ was dispensed onto the polydopaminecoated surface from a height of $2 \mathrm{~cm}$ to determine the static contact angle. After that, the needle was brought down into the drop from the top center. Advancing and receding contact angles were measured by increasing $10 \mu \mathrm{L}$ (growing phase) and then decreasing $15 \mu \mathrm{L}$ (contracting phase) of the drop volume at a rate of $100 \mu \mathrm{L} / \mathrm{min}$ to ensure that the three phase boundary line moved over the surface. A period of 20 sec, called the relaxing phase, was waited between the growing phase and the contracting phase. The images of the drop were recorded and analyzed by a custom-developed LabVIEW (version 8.5, National Instruments) program in terms of contact angle, drop volume, and drop contact radius as a function of time. All measurements were performed in ambient air at the room temperature.

\subsection{Contact Adhesion of Polydopamine Thin Films}

Contact adhesion measurements were carried out using a custom-made micro-indentation system that is comprised of a linear stage (Newport MFA-CC), a 25-gram force transducer (Transducer Techniques GS0-25), and an inverted optical microscope (Omano OMM300T) and controlled by a custom-developed LabVIEW (version 8.5, National Instruments) program. Hemispherical PDMS tips were brought into contact with flat sheets of PDMS coated on glass, as shown in Fig. (1B), at a speed of $0.1 \mu \mathrm{m} / \mathrm{sec}$ until a preload force of $0.5 \mathrm{mN}$ was detected, held in contact at the preload for $5 \mathrm{~min}$, and then separated at the same speed. Three sets of tests, uncoated PDMS tip on PDMS substrate (PDMSPDMS), PDMS tip on polydopamine-coated PDMS substrate (PDMS-PDMS(D)), and polydopamine-coated PDMS tip on polydopamine-coated PDMS substrate (PDMS(D)-PDMS (D)), were conducted in ambient air at the room temperature. Force, displacement, and time information were recorded.

\subsection{Pull-Off Tests of Dopamine Adhesive Bonds/Joints}

Pull-off tests of aluminum studs joined by dopamine aqueous solutions on glass and PDMS substrates were performed to determine the adhesive bonding strength of polydopamine. PDMS substrates were pre-coated with dopamine solution at $2 \mathrm{mg} / \mathrm{mL}$ for 1 hour to reduce surface hydrophobicity. Aluminum studs, $4 \mathrm{~g}$ in weight and $12.7 \mathrm{~mm}$ in base diameter, were placed on top of the substrates with 2 $\mu \mathrm{L}$ of dopamine solution in between, as shown in Fig. (1C). The joints were placed in ambient air at the room temperature for 1 hour then at $90{ }^{\circ} \mathrm{C}$ for 1 hour to allow dopamine to polymerize and dry before testing. The bond strength was determined using a Texture Analyzer (Texture Technologies Corp. TA.XT.plus) at a speed of $0.1 \mathrm{~mm} / \mathrm{sec}$.

\section{RESULTS AND DISCUSSION}

\subsection{Wettability Characterization}

Good wetting of adhesives on substrate surfaces is the prerequisite for successful bonding or joining. The wettability of polydopamine-coated PDMS surfaces was investigated by water contact angle measurements. The static contact angle of deionized water drop on polydopaminecoated PDMS surface was measured to be $65^{\circ}$, revealing the hydrophilic nature of self-polymerized dopamine films. This static contact angle is consistent with the value reported in literature [11] suggesting the PDMS substrate was completely covered by a layer of polydopamine. Note that the PDMS surface is hydrophobic, having a water contact of $105^{\circ}$. To obtain further insights on the properties of polydopamine-coated surface, we designed and performed dynamic contact angle measurements by continuously enlarging and subsequently reducing the size of water drop through an embedded needle. Fig. (2A) shows typical optical images of initial, advancing, and receding water drops for static and dynamic contact angle measurements. Fig. (2B) plots the contact angle, volume, and radius of the contact area of the drop on the surface as a function of time. We noticed that the initial contact angle in the dynamic measurement was same as the static contact angle, suggesting a negligible effect of the embedded needle on contact angle. The contact angle of the drop in the growing state that reached a steady-state value after $7 \mathrm{sec}$ is defined as the advancing contact angle $\theta_{a}$, which was about 5 degrees higher than the static one. There was a slight delay between the volume increase and the area increase, called the contact line "pinning" effect [31]. As the drop stopped growing, the contact angle relaxed and remained constant at an angle about 4 degrees higher than the initial angle, perhaps due to the increase of volume and the pinning effect. In the contracting region, a more significant contact line "pinning" effect was observed; this effect caused the contact angle to decrease with the volume while the radius remained constant. The lowest contact angle, occurred at the moment the contact line started to move, is defined as the receding contact angle $\theta_{\mathrm{r}}$, which is $10^{\circ}$. The contact angle hysteresis, i.e. $\theta_{\mathrm{a}}-\theta_{\mathrm{r}}$ was determined to be $60^{\circ}$. The low receding angle and large hysteresis suggested the formation of a hydration layer or bound water layer upon contacting with water. This is reasonable considering the amine and hydroxyl groups of dopamine are capable to reorient and form hydrogen bonds with water molecules. Thus, we suspect that the surface of polydopamine coating layer had not been completely polymerized; a large amount of free amine and hydroxyl groups existed at the surface.

\subsection{Contact Adhesion Properties of Polydopamine- Coated Surfaces}

The adhesion properties of polydopamine thin films were studied in terms of self-adhesion and adhesion to PDMS surfaces. Fig. (3A) shows a typical force $v s$ displacement curve for the contact adhesion between two polydopaminecoated surfaces, characterized by a negative tensile force and a loading-unloading hysteresis. The surface force induced "jump-in" phenomenon was not observed in the selfadhesion tests suggesting that either the surface was rigid or rough. For quantitative analyses, we defined three characteristic parameters extracted from the force vs displacement curves: (a) the pull-off or separation force called the adhesive force $F_{s}=-L_{s}$, (b) the adhesion hysteresis $\mathrm{W}_{\mathrm{h}}$ (i.e. the energy loss during the loading-unloading cycles), and (c) the indentation depth or displacement $\delta_{\text {ind }}$ at the applied preload, which is related to surface compliance.

Fig. (3B) is a comparison of these three parameters obtained from the indentation tests between (a) two bare PDMS surfaces as a control, (b) polydopamine-coated and PDMS surfaces, and (c) two polydopamine-coated surfaces. The indentation depth $\delta_{\text {ind }}$ declined consistently in the order of PDMS-PDMS, PDMS-PDMS(D), and PDMS(D)PDMS(D) suggesting the polydopamine thin film is more 
(A)

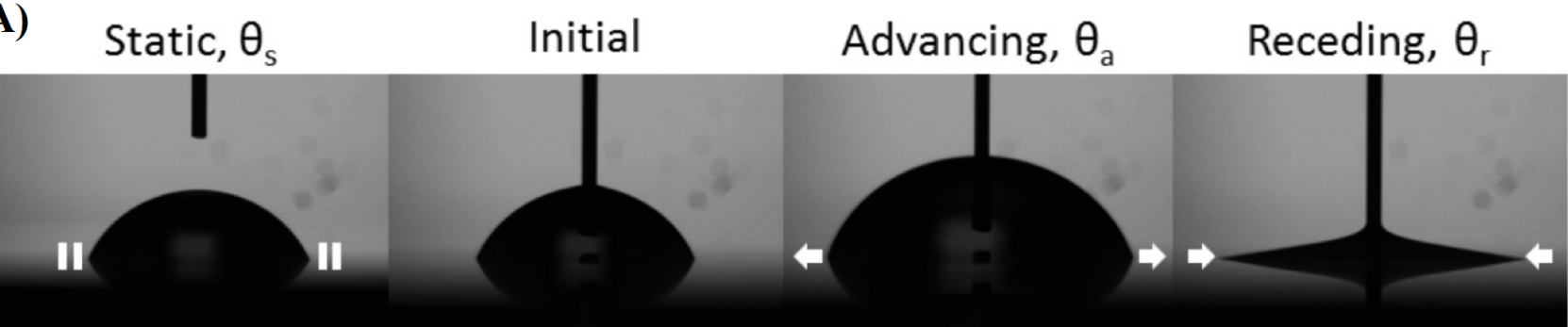

(B)

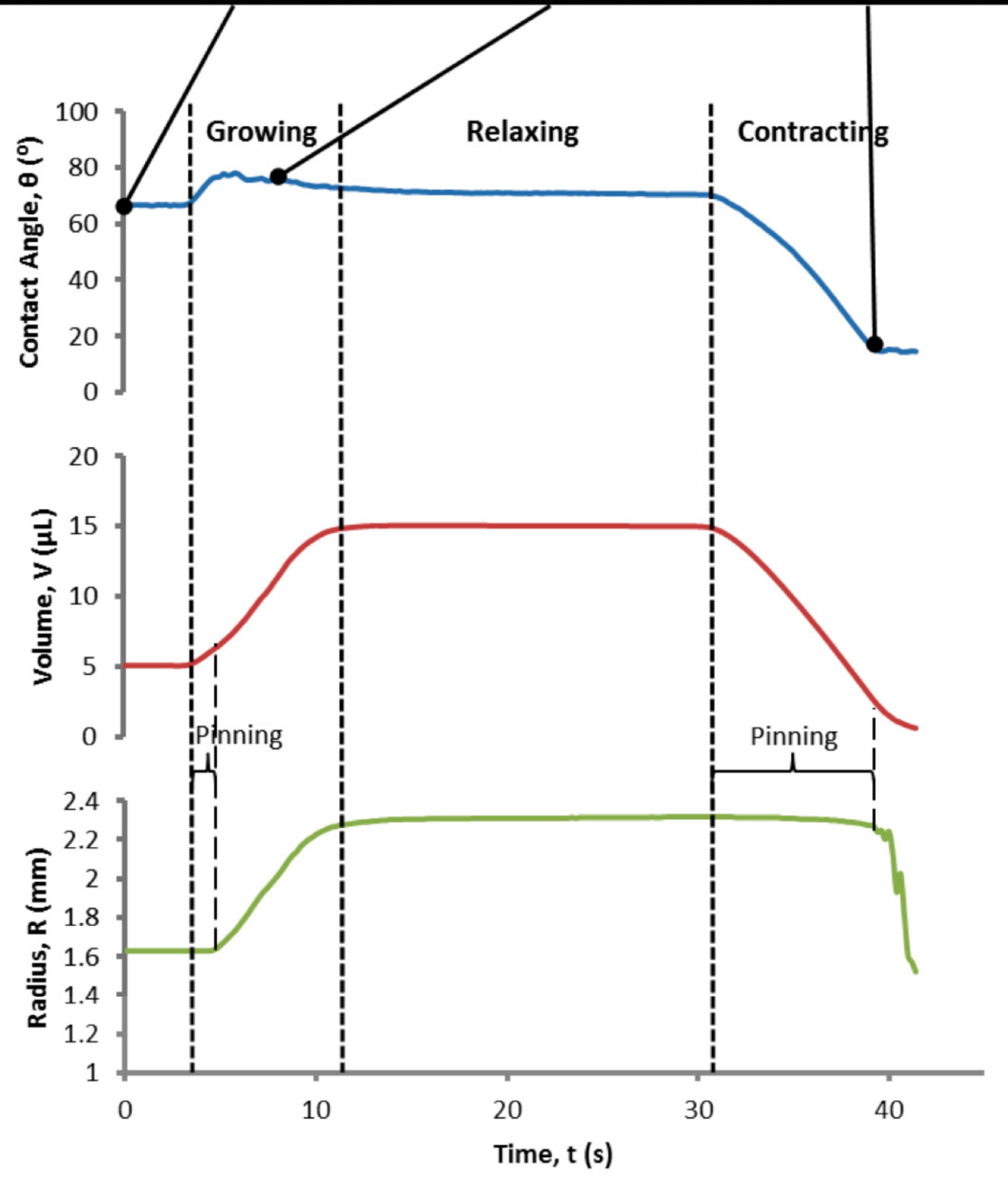

Fig. (2). Wettability characterization: (A) typical optical images of static and dynamic contact angles and (B) plots of contact angle, drop volume and drop contact radius vs time.

rigid than the PDMS surface. As for the adhesive force $F_{s}$, the asymmetric contacts between PDMS and PDMS(D) have the highest value while the contacts between PDMS(D) and PDMS(D) have the lowest value. The comparison in the hysteresis parameter shows a much more pronounced difference between the asymmetric contacts and the other two symmetric contacts. It is interesting to notice that the polydopamine surface seemed inert to itself but active to the PDMS surface. We suspect that there might be specific interactions between polydopamine and PDMS considering that some amine and hydroxyl groups on the former surface might not be polymerized (as revealed from the large contact angle hysteresis) and were able to actively interact with the latter surface. The reason for the observed low self-adhesion of polydopamine-coated surface is not known. Ongoing indepth characterizations of the polydopamine coating in terms of surface composition and topography will be able to reveal more insights into this observation. 
(A)

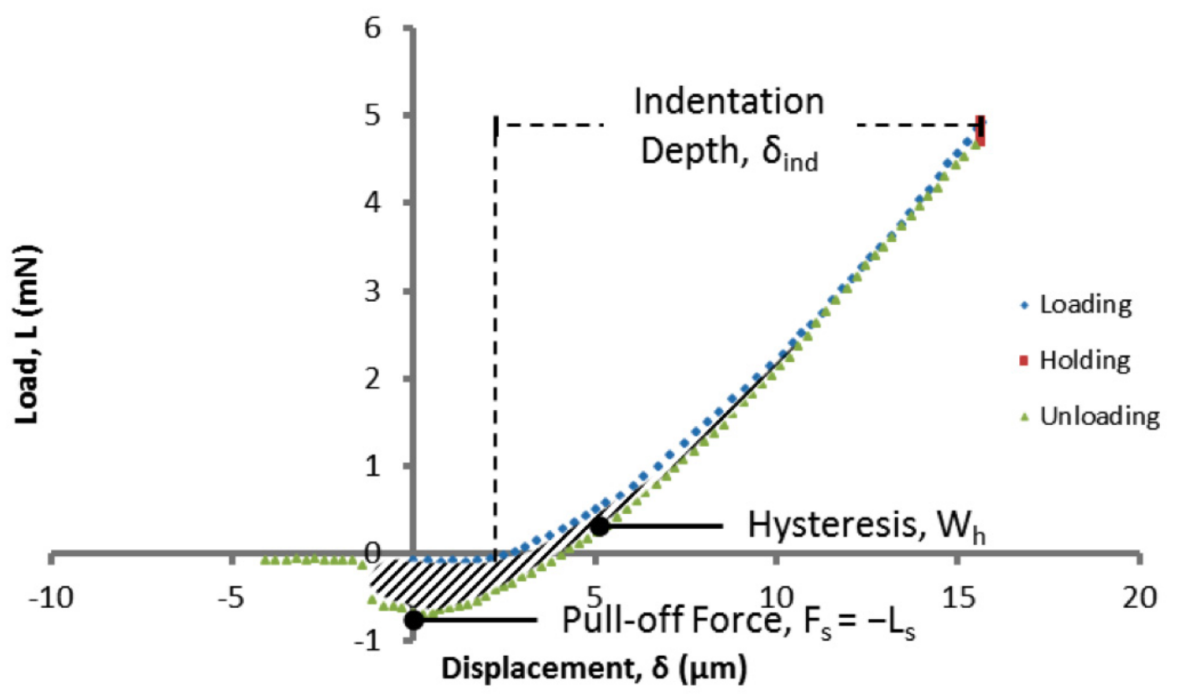

(B)

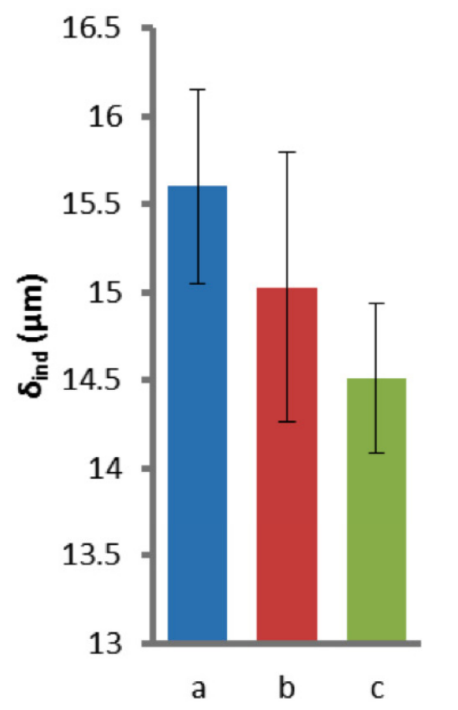

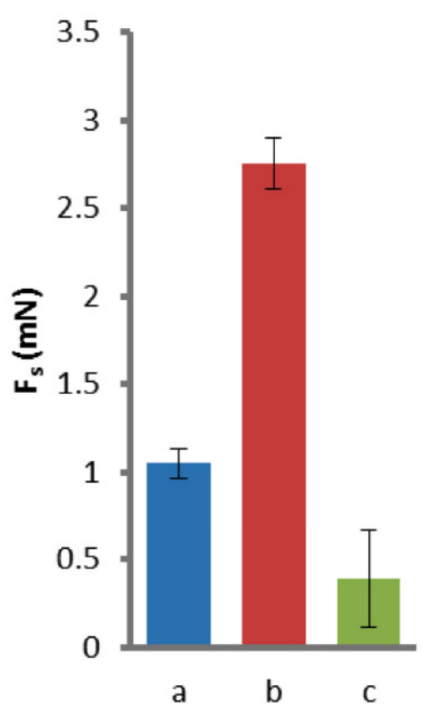

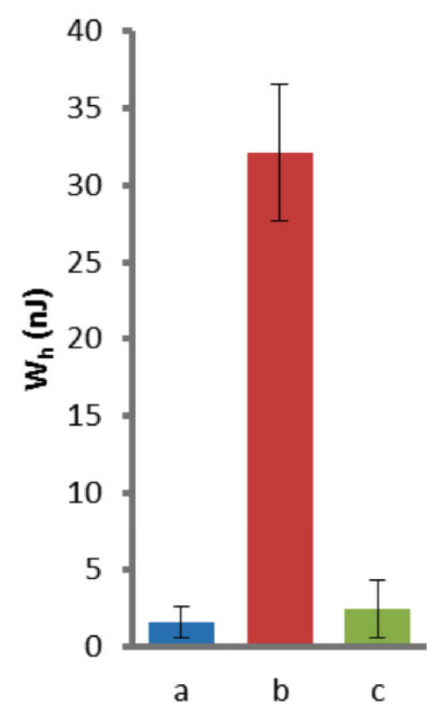

Fig. (3). Contact adhesion properties of polydopamine-coated surfaces. (A) typical load vs displacement curve and three characteristic parameters: indentation depth $\delta_{\text {ind }}$, pull-off force $\mathrm{F}_{\mathrm{s}}$, and hysteresis $\mathrm{W}_{\mathrm{h}}$, and $(\mathbf{B})$ comparison of the three parameters for the contacts between (a) PDMS and PDMS, (b) PDMS and PDMS(D), and (c) PDMS(D) and PDMS (D).

\subsection{Dopamine Adhesive Bonding}

The adhesive bonding behavior and the practicality of joining with dopamine solutions of different concentrations were investigated by using the solutions to join aluminum studs to two different flat substrates: hydrophilic glass and hydrophobic PDMS elastomer. We noticed that the dopamine solution could not directly be used to join the Al and PDMS because PDMS is hydrophobic and the solution was squeezed out during bonding. We found a pretreatment of PDMS with dopamine necessary for a successful bonding process. As shown in the wettability studies, the surface of PDMS can be changed from hydrophobic to hydrophilic after coating with a thin layer of polydopamine. We utilized this finding and brought the contact angle of PDMS down to $95^{\circ}$ with 1 hour of pretreatment to avoid the squeeze-out of dopamine solutions. The bonded areas were examined under an optical microscope; circular shapes were observed with a diameter of $5 \mathrm{~mm}$ for $\mathrm{Al} / \mathrm{glass}$ and $10 \mathrm{~mm}$ for Al/PDMS.
Fig. (4A) shows the typical force vs displacement curve when pulling bonded substrates apart in tensile mode. The maximum force, called the pull-off force, was normalized by the bonded area to give the adhesive bonding strength in $\mathrm{N} / \mathrm{m}^{2}$. The detached surfaces were examined under an optical microscope showing remains of dopamine adhesive on both surfaces. Thus, the failure of adhesive bonds happened mostly within the polydopamine layer revealing that the bonds formed between dopamine and its substrate surface is strong. This is consistent with the observation of the low self-adhesion of polydopamine surfaces. Fig. (4B) shows the bonding strength as a function of dopamine concentrations on glass and on PDMS substrate. It can be seen that the dopamine bonding of PDMS and $\mathrm{Al}$ is significantly different from that of glass and Al. There was no adhesion between bare Al and glass; the adhesive strength increased with dopamine concentration and leveled off at a concentration of $5 \mathrm{mg} / \mathrm{mL}$ or a surface coverage of $0.51 \mathrm{~g} / \mathrm{m}^{2}$. For the combination of PDMS and Al, there was a finite adhesion 
(A)

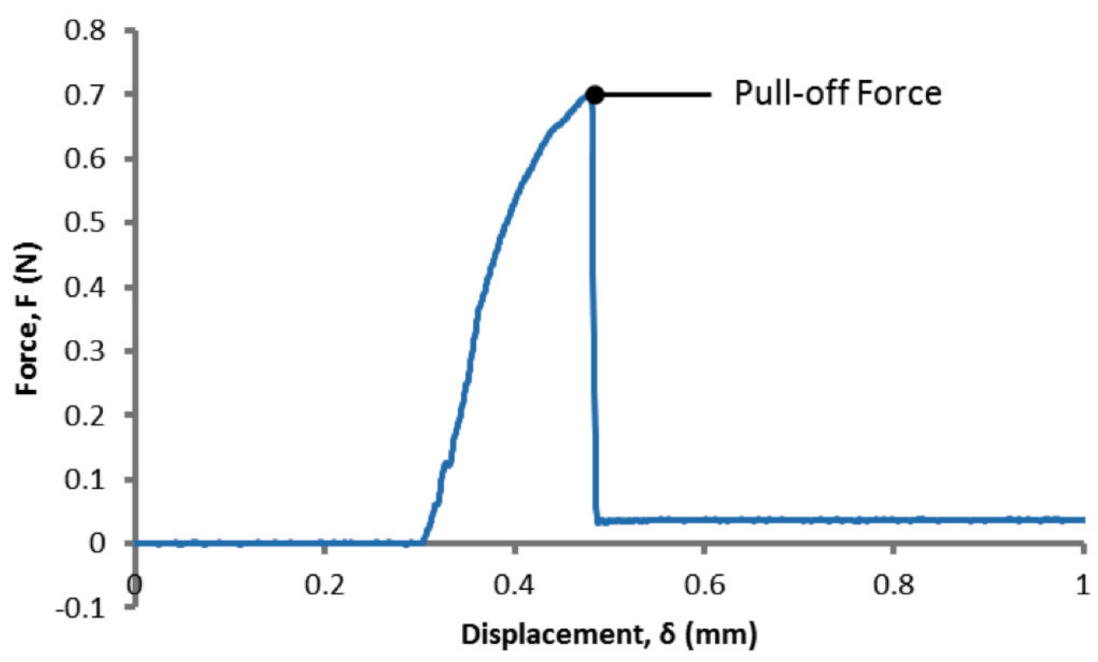

(B)

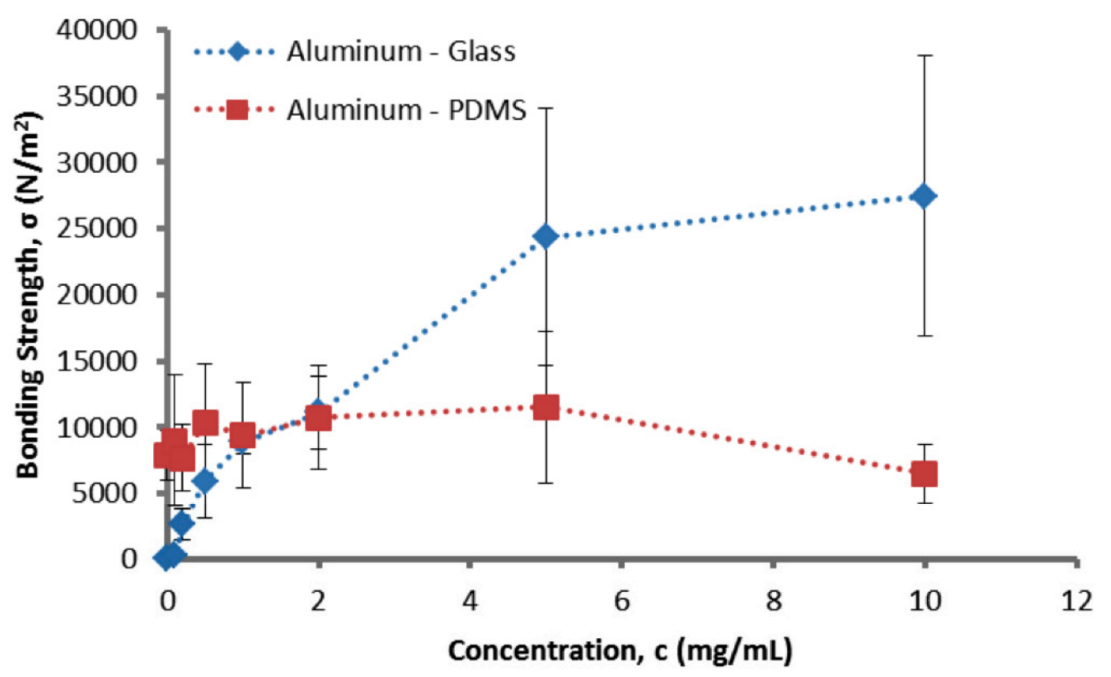

Fig. (4). Dopamine adhesive bonding. (A) typical force displacement curve for the pull-off measurement and (B) plot of adhesive bonding strength $v s$ dopamine concentrations.

between the bare surfaces; adding dopamine has a negligible effect on the adhesive strength at low concentrations but a detrimental effect when the concentration is above $5 \mathrm{mg} / \mathrm{mL}$ or a surface coverage of $0.13 \mathrm{~g} / \mathrm{m}^{2}$.

To acquire further insights into the adhesive bonding of the polydopamine layer, we carefully examined polydopamine-coated surfaces using the Veeco Optical Profiling System. Fig. (5) shows typical images: no cracks were seen on the polydopamine-coated glass surfaces (Fig. 5A), but numerous micron-sized cracks were seen on the polydopamine-coated PDMS surface (Fig. 5B). Furthermore, when we coated a thick layer of polydopamine on PDMS and slightly bent it, we found visible macron-sized cracks formed in the polydopamine films that partly delaminated from the substrate (Fig. 5C). The cracks revealed the brittle nature of the dry polydopamine film. Mismatch of the elastic modulus between the rigid polydopamine film and its soft substrate might be the reason of forming cracks, rendering the polydopamine bonding of PDMS and Al ineffective. We may conclude that the brittle nature of polydopamine thin film limits its cohesive strength which in turn limits the overall bonding strength.

\section{CONCLUSIONS}

Three types of experimental studies (water contact angle, contact adhesion, and adhesive bonding) had been performed to investigate the adhesion behavior of polydopamine thin films coated on or bonded between inorganic and polymer substrates. Polydopamine-coated polydimethylsiloxane surfaces had a static contact angle of $65^{\circ}$, suggesting a complete surface coverage by a layer of polydopamine. Wettability of the polydopamine-coated surface was further characterized by dynamic water contact angle measurements, revealing a large contact angle hysteresis of about $60^{\circ}$ between the advancing and receding angles. The low receding contact angle suggested the formation of a hydration layer when exposed to water. Polydopamine-coated surfaces in air are relatively inert and have a low self-adhesion compared with the uncoated PDMS surfaces, reflecting the non-conformal, glassy nature of the polydopamine layer. The asymmetric contact between the polydopamine-coated surface and PDMS surface showed the highest adhesion force and hysteresis. The dopamine solutions was found able to bond two rigid surfaces (Al and glass) and the adhesive bonding strength increased with the amount of dopamine and 

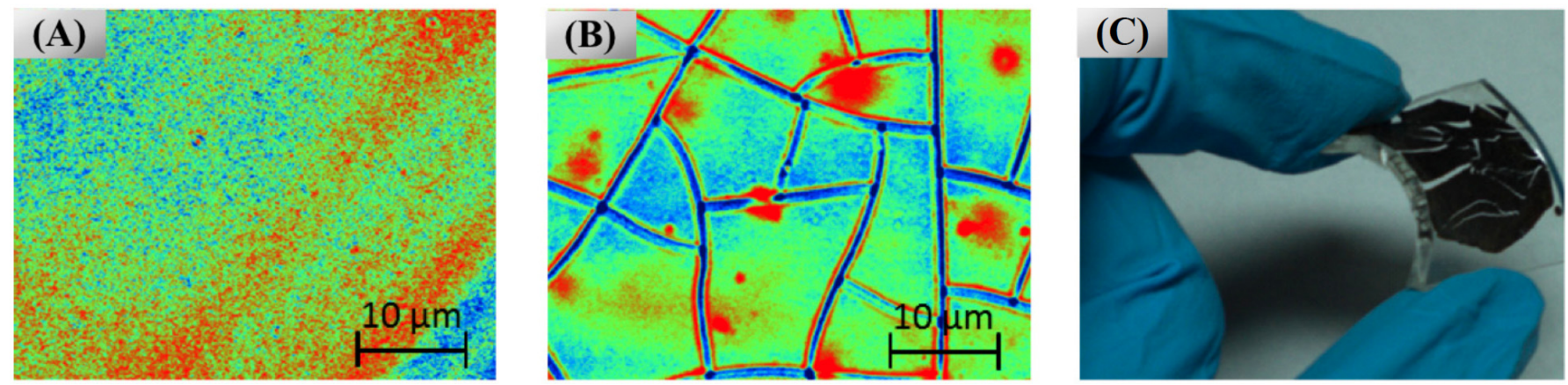

Fig. (5). Optical images of (A) polydopamine-coated glass surface, (B) polydopamine-coated PDMS surface by Veeco optical profiling system, and (C) bent polydopamine-coated PDMS.

saturated at $5 \mathrm{mg} / \mathrm{mL}$. The direct joining of Al and PDMS using dopamine solution was challenging because of the hydrophobic nature of PDMS; a pre-coating of dopamine on PDMS was found useful. There were no significant bonding strength enhancements in the PDMS/Al system. In summary, the present study in elucidating the surface and adhesion behavior of polydopamine revealed that dopamine is able to coat plastic, ceramic and metal surfaces, and join or bond rigid substrates but might not be suitable for joining soft or flexible parts as polydopamine films are glassy.

\section{ACKNOWLEDGEMENTS}

The authors would like to thank the Natural Science and Engineering Research Council of Canada (NSERC) for the financial support. The authors also acknowledge Mr. Yougun Han for the assistance with the instrumentation of wettability and contact adhesion measurements.

\section{REFERENCES}

[1] Lee H, Scherer NF, Messersmith PB. Single-molecule mechanics of mussel adhesion. Proc Natl Acad Sci USA 2006; 103(35): 12999-3003.

[2] Lee H, Dellatore SM, Miller WM, Messersmith PB. Musselinspired surface chemistry for multifunctional coatings. Science 2007; 318(5849): 426-30.

[3] Lee H, Lee Y, Statz AR, Rho J, Park TG, Messersmith PB. Substrate-independent layer-by-layer assembly by using musseladhesive-inspired polymers. Adv Mater 2008; 20(9): 1619-23.

[4] Lee H, Rho J, Messersmith PB. Facile conjugation of biomolecules onto surfaces via mussel adhesive protein inspired coatings. Adv Mater 2009; 21(4):431-4.

[5] Kang S, Elimelech M. Bioinspired single bacterial cell force spectroscopy. Langmuir 2009; 25(17): 9656-9.

[6] Ouyang R, Lei J, Ju H. Artificial receptor-functionalized nanoshell: facile preparation, fast separation and specific protein recognition. Nanotechnology 2010; 21(18): 185502.

[7] Ye W, Wang D, Zhang H, Zhou F, Liu W. Electrochemical growth of flowerlike gold nanoparticles on polydopamine modified ITO glass for SERS application. Electrochim Acta 2010; 55(6): 2004-9.

[8] Zhou W, Tang S, Yao Q, Chen F, Yang H, Wang X. A quartz crystal microbalance sensor based on mussel-inspired molecularly imprinted polymer. Biosens Bioelectron 2010; 26(2): 585-9.

[9] Postma A, Yan Y, Wang Y, Zelikin AN, Tjipto E, Caruso F. Selfpolymerization of dopamine as a versatile and robust technique to prepare polymer capsules. Chem Mater 2009; 21(14): 3042-4.

[10] $\mathrm{Ku} \mathrm{SH}$, Lee JS, Park CB. Spatial control of cell adhesion and patterning through mussel-inspired surface modification by polydopamine. Langmuir 2010; 26(19): 15104-8.

[11] $\mathrm{Ku} \mathrm{SH}$, Ryu J, Hong SK, Lee H, Park CB. General functionalization route for cell adhesion on non-wetting surfaces. Biomaterials 2010; 31(9): 2535-41.

[12] Cui J, Wang Y, Postma A, Hao J, Hosta-Rigau L, Caruso F. Monodisperse polymer capsules: tailoring size, shell thickness, and hydrophobic cargo loading via emulsion templating. Adv Funct Mater 2010; 20(10): 1625-31.

[13] Fei B, Qian B, Yang Z, et al. Coating carbon nanotubes by spontaneous oxidative polymerization of dopamine. Carbon 2008 46(13): 1795-7.

[14] Ben-Valid S, Botka B, Kamarás K, Zeng A, Yitzchaik S. Spectroscopic and electrochemical study of hybrids containing conductive polymers and carbon nanotubes. Carbon 2010; 48(10): 2773-81.

[15] $\mathrm{Hu} \mathrm{H}, \mathrm{Yu}$ B, Ye Q, Gu Y, Zhou F. Modification of carbon nanotubes with a nanothin polydopamine layer and polydimethylamino-ethyl methacrylate brushes. Carbon 2010; 48(8): 2347-53.

[16] Ye W, Hu H, Zhang H, Zhou F, Liu W. Multi-walled carbon nanotube supported $\mathrm{Pd}$ and $\mathrm{Pt}$ nanoparticles with high solution affinity for effective electrocatalysis. Appl Surf Sci 2010; 256(22): 6723-8.

[17] Zhang M, Zhang X, He X, Chen L, Zhang Y. Preparation and characterization of polydopamine-coated silver core/shell nanocables. Chem Lett 2010; 39(6): 552-3.

[18] Bernsmann F, Richert L, Senger B, et al. Use of dopamine polymerisation to produce free-standing membranes from (PLLHA)n exponentially growing multilayer films. Soft Matter 2008; 4(8): $1621-4$

[19] Li B, Liu W, Jiang Z, Dong X, Wang B, Zhong Y. Ultrathin and stable active layer of dense composite membrane enabled by poly(dopamine). Langmuir 2009; 25(13): 7368-74.

[20] Ou J, Wang J, Liu S, Zhou J, Yang S. Self-assembly and tribological property of a novel 3-layer organic film on silicon wafer with polydopamine coating as the interlayer. J Phys Chem C 2009; 113(47): 20429-34.

[21] Xi Z, Xu Y, Zhu L, Wang Y, Zhu B. A facile method of surface modification for hydrophobic polymer membranes based on the adhesive behavior of poly(DOPA) and poly(dopamine). J Membr Sci 2009; 327(1-2): 244-53.

[22] Bernsmann F, Ersen O, Voegel J, Jan E, Kotov Na, Ball V. Melanin-containing films: growth from dopamine solutions versus layer-by-layer deposition. Chem Phys Chem 2010; 48109: 3299 305.

[23] Jiang J, Zhu L, Li X, Xu Y. Surface modification of PE porous membranes based on the strong adhesion of polydopamine and covalent immobilization of heparin. J Membr Sci 2010; 364(1-2): 194-202.

[24] McCloskey BD, Park HB, Ju H, Rowe BW, Miller DJ, Chun BJ. Influence of polydopamine deposition conditions on pure water flux and foulant adhesion resistance of reverse osmosis, ultrafiltration, and microfiltration membranes. Polymer 2010; 51(15): 3472-85.

[25] Ryu J, Ku SH, Lee H, Park CB. Mussel-inspired polydopamine coating as a universal route to hydroxyapatite crystallization. Adv Funct Mater 2010; 20(13): 2132-9.

[26] Xing B, Yin X. Novel poly-dopamine adhesive for a halloysite nanotube-Ru(bpy)(3)2+ electrochemiluminescent sensor. PloS One 2009; 4(7): e6451.

[27] Xu LQ, Yang WJ, Neoh K, Kang E, Fu GD. Dopamine-induced reduction and functionalization of graphene oxide nanosheets. Macromolecules 2010; 43(20): 8336-9. 
[28] Bernsmann F, Ponche A, Ringwald C, et al. Characterization of dopamine-melanin growth on silicon oxide. J Phys Chem C 2009; 113(19): 8234-42.

[29] Harrington MJ, Masic A, Holten-Andersen N, Waite JH, Fratzl P. Iron-clad fibers: a metal-based biological strategy for hard flexible coatings. Science 2010; 328(5975): 216-20.
[30] Wei Q, Zhang F, Li J, Li B, Zhao C. Oxidant-induced dopamine polymerization for multifunctional coatings. Polym Chem 2010; 1(9): 1430-33.

[31] De Gennes PG. Wetting: statics and dynamics. Rev Mod Phys 1985; 57(3): 827-63.

(C) Yang and Zhao; Licensee Bentham Open.

This is an open access article licensed under the terms of the Creative Commons Attribution Non-Commercial License (http://creativecommons.org/licenses/ by$\mathrm{nc} / 3.0 /$ ) which permits unrestricted, non-commercial use, distribution and reproduction in any medium, provided the work is properly cited. 